\title{
Guest Editorial: Marine Robotics and Control Systems
}

This special issue is devoted to the topic of marine robot's control, intelligence, and perception. In terms of the mobility platform, a marine robot is challenging due to the restriction of sensing and moving environment, water surface, or underwater. In terms of marine vehicle control, there are many rooms to improve. This special issue deals with the control strategy of a surface vessel and an underwater vehicle. Surface and underwater perception is the key to automate the marine system. Optical vision is strictly limited in underwater and sonar data; few available sensing methods have poor $\mathrm{S} / \mathrm{N}$ with low resolution. Electromagnetic or electric field-based sensing is very challenging. This special issue provides representative solutions for the sensing and perception in marine sensing. Localization and the multi-agent system are also important issues of marine robots. This issue deals with the precision ASV navigation method and optimal multi-ASV operation strategy. Seabed or underwater objects' 3D mapping is also a critical application of marine robots. Notably, most papers of this special issue have sea-trial with actual marine robot/vehicle. These studies could provide solid lessons for the marine robot's study.

The first paper entitled "Realistic Sonar Image Simulation Using Deep Learning for Underwater Object Detection" proposed the underwater sonar's emulator. This paper focused on the background noise as well as the foreground object. The background noise is critical to generate a reliable simulator. It is the most significant contribution of this study.

The next paper entitled "Development of an Autonomous Surface Vehicle and Performance Evaluation of Autonomous Navigation Technologies" contains two critical issues; how to develop a precision navigation purpose ASV and the how-to realize path-following navigation in the field. Acoustic-based and terrain-based navigation methods were proposed and conducted their performance.

The third paper entitled "Collaborative Mission and Route Planning of Multi-vehicle Systems for Autonomous Search in Marine Environment" deals with a multi-agent system. This study proposes the efficient deployment of a multiple ASV troop. Depending on the situation, obstacles, and agents' positions, efficient collaborative path-planning, and task allocation methods were addressed.

The following paper entitled "Control Allocation for Double-ended Ferries with Full-scale Experimental Results" proposed a novel control allocation algorithm for the double-ended ferries. The author proposed a cost function and bounds for that. The real-time performance of the proposed algorithm is demonstrated in a simulation study and full-scale experiments.

The fifth paper entitled "Three-dimensional Visual Mapping of Underwater Ship Hull Surface Using Piecewise-planar SLAM" proposed an optical vision-based visual mapping method for 3D reconstruction of underwater objects using a monocular camera as a primary mapping sensor. The main idea of the proposed approach is to model the moderately curved hull surface as a combination of piecewise-planar panels and to generate a global map by aligning the local images in a two-dimensional reference frame and correcting them appropriately to reflect the information of perspective projections of the 3D panels. The proposed method is demonstrated using a dataset obtained in a field experiment with a full-scale ship in a real sea environment.

The next paper entitled "Analysis of Electromagnetic Waves Attenuation for Underwater Localization in Structured Environments" deals electromagnetic (EM) sensing for underwater localization. Near or inside of the structured environment, EM is few available sensing methods in underwater. The authors analyzed EM wave attenuation depending on the position and materials for the accurate ranging or positioning of marine robots/ underwater sensing.

The working paper entitled "3D Reconstruction Using Two Sonar Devices in a Monte-Carlo Approach for AUV Application" proposed sonar-fusion based 3D reconstruction for AUV navigation. Two image sonars are mounted perpendicularly, and their data have complementary characteristics. Fused sonar data significantly improved the accuracy of the 3D reconstruction. 
The eighth paper entitled "Tracking a Sea Turtle by an AUV with a Multibeam Imaging Sonar: Toward Robotic Observation of Marine Life" is a unique study; the world's first autonomous tracking of a living turtle using an underwater robot. The AUV recognize a turtle using a sonar image and controls the path for following. For the reliable and efficient recognition of the turtle using sonar imagery, CNN based recognition method was implemented to the AUV and actual turtle following test was conducted.

The following paper entitled "Underwater Image Dehazing via Unpaired Image-to-image Translation" proposed Generative Adversarial Networks (GAN) based underwater imagery enhancement method. In this paper, the authors constituted unpaired image translation frameworks into image restoration. The proposed method utilizes multiple cyclic consistency losses that capture image characteristics and details of underwater images. For the validation, comparison with the conventional methods have been carried out.

The last paper entitled "Adaptive Non-singular Terminal Sliding Mode Control for an Unmanned Underwater Vehicle: Real-time Experiments" focuses on the design of a robust-adaptive control algorithm for a 4DOF UUV. The proposed strategy is based in a Non-Singular Terminal Sliding Mode Control with adaptive gains, where the proposed adaptation mechanism ensures that the gains remain bounded. For the validation and evaluation of the control performance, actual experiments were conducted with a ROV.

\section{Acknowledgments}

The guest editors would like to express sincere thanks to all the authors for their significant contributions to Special Issue on Marine Robotics and Control Systems and many reviewers who provided many valuable review comments to improve the quality of each contributed manuscript. We do also appreciate the great supports by the Editor-in-Chief of IJCAS, Prof. Keum-Shik Hong, and editorial office manager Ms. Jinyoung You from the planning through the publication of this particular issue.

\section{Guest Editors}

\section{Son-Cheol Yu}

Professor at Pohang University of Science and Technology (POSTECH), Korea

e-mail: sncyu@ postech.ac.kr

Antonio M. Pascoal

Professor at University of Lisbon, Portugal

e-mail: antonio@isr.tecnico.ulisboa.pt

Jinwhan Kim

Professor at Korea Advanced Institute of Science and Technology (KAIST), Korea

e-mail: jinwhan@kaist.ac.kr 